\title{
Study on Optimizing High-Gradient Magnetic Separation-Part 1: Improvement of Magnetic Particle Retention Based on CFD Simulations
}

\author{
Yonas S. Shaikh, Christian Seibert, Percy Kampeis \\ Trier University of Applied Sciences, Environmental Campus Birkenfeld, Institute for Biotechnical Process \\ Design, Birkenfeld, Germany \\ Email:y.shaikh@umwelt-campus.de
}

Received 24 March 2016; accepted 28 May 2016; published 31 May 2016

Copyright (C) 2016 by authors and Scientific Research Publishing Inc.

This work is licensed under the Creative Commons Attribution International License (CC BY). http://creativecommons.org/licenses/by/4.0/

c) (i) Open Access

\begin{abstract}
The introduction of functionalized magnetizable particles for the purification of enzymes or for the multi-use of pre-immobilized biocatalysts offers a great potential for time and cost savings in biotechnological process design. The selective separation of the magnetizable particles is performed for example by a high-gradient magnetic separator. In this study FEM and CFD simulations of the magnetic field and the fluid flow field within a filter chamber of a magnetic separator were carried out, to find an optimal separator design. The motion of virtual magnetizable particles was calculated with a one-way coupled Lagrangian approach in order to test many geometric and parametric variations in reduced time. It was found that a flow homogenisator smoothed the fluid flow, so that the linear velocity became nearly equal over the cross section in the direction of flow. Furthermore the retention of magnetizable particles increases with a high total edge length within the filter matrix.
\end{abstract}

\section{Keywords}

Computational Fluid Dynamics, Finite Element Method, Magnetic Particles, High-Gradient Magnetic Separator, Magnetic Filtration, Biocatalysis, Enzyme Purification

\section{Introduction}

The use of functionalized magnetizable particles in combination with a high-gradient magnetic separator is an

How to cite this paper: Shaikh, Y.S., Seibert, C. and Kampeis, P. (2016) Study on Optimizing High-Gradient Magnetic Separation-Part 1: Improvement of Magnetic Particle Retention Based on CFD Simulations. World Journal of Condensed Matter Physics, 6, 123-136. http://dx.doi.org/10.4236/wjcmp.2016.62016 
efficient way to perform a solid/solid/liquid separation in one step. Functionalized magnetizable particles with a diameter in the nano- or micron-range for various applications are described in some publications [1]-[6]. Hereby the single-step purification of enzymes or the multiple uses of pre-immobilized biocatalysts on magnetizable particles showed a great potential as alternatives to conventional processes.

Within the total number of publications regarding the usage of magnetizable particles and magnetic separation in bioengineering, only a little fraction is focused on the design of the separator itself. The reason for this could be that the separation in bench-scale was very simple and practicable with a permanent magnet. Normally, when the separation takes place in milliliters, either the magnet or the suspension container is removed manually for resuspension of the magnetic particle deposits. But when magnetic separation is conducted for several liters, a magnetic filter is required. The fluid is pumped through a filter chamber in which a matrix insert of highly magnetizable stainless steel is placed [7]-[10]. Hereby the magnetic field is applied by permanent magnets or electromagnets. The permanent magnets have the advantages that they have no power consumption and show no thermal load up. The disadvantage is the always active magnetic field which cannot be easily switched off as with an electromagnet. To overcome this disadvantage, a magnetic separator with a switchable magnetic field within the air gap of the yoke is published by Hoffmann et al. [11]. The separator was used in several studies for the separation of biomolecules from fermentations and biocatalytic processes [11]-[17].

Until now the magnetic yoke of the separator was not varied and analyzed for optimization. The air gap of the magnetic yoke can be varied with different steel pole plates, which decrease the distance of the pole surfaces to each other. Herewith the separator could be modified for the process requirements. In this study the distance and the surface area of the pole plates were varied to determine the effect of different geometries on the magnetic flux density in the middle of the air gap.

The magnetizable matrix inserts in the filter chamber were mainly made of stainless steel wool. Hereby the rinsing of the filter chamber was not efficient and the optimization of the filter matrix was the focus of some studies [16] [18]-[22]. But within these studies the inlet and the resulting fluid flow profile at the start of the separator were not varied and described. It was found that the separation of magnetizable particles took place in the front part of the filter chamber. This is a crucial factor affecting the overall performance of magnetic separation [23] [24].

Therefore, in this study the optimization of a high-gradient magnetic separator was performed using Finite Element Method (FEM) and Computational Fluid Dynamic (CFD) simulations. On this basis, trajectories of virtual magnetizable particles were calculated to quantify the effects of the geometric variations. The use of a flow homogenisator was tested to quantify the distribution of the linear fluid velocity and the particle distribution on a cross-section near the filter matrix in the filter chamber. The retention of magnetizable particles on filter matrices with different geometries was tested to find the optimal cutouts in the filter plates.

Previous studies on the behavior of magnetizable particles in a magnetic field and a fluid flow field were based on different approaches. A simple and easy to compute approach is the Lagrangian one-way coupled simulation. Hereby the magnetic field and the fluid flow field are calculated at first and the Newtonian particle motion is calculated on the basis of the two previous fields. The particles do influence neither the fluid flow field nor the magnetic field [25]. With the two-way coupling approach the magnetic field or the fluid flow field is influenced by the location of the particle load. Previous studies of Khashan et al. [26] [27] include a non-pertubated magnetic field but a fluid flow field interacting with the particle concentration in the suspension. Here it was shown that for very small magnetizable particles, the magnetophoresis migration is dominant over molecular diffusion. When particle-particle interaction is included, the separation rate becomes better due to particle clustering [28] [29]. The study of Lindner et al. [30] showed that the introduction of a Discrete Element Method enables the analysis of the shape of magnetic particle deposits on single wires. In general, the design of a magnetic filter was described by Ravnik et al. [31] to be of a short length in the flow direction, since the number of collected particles decreases with the distance to the inlet. It is suggested to operate multiple parallel separation units. In a study of magnetic particle separation with a micro-channel and a laminar flow of water, it was found by Khashan et al. [32] that the one-way coupling marginally under-predicts the capture efficiency compared to the two-way coupling. The Lagrangian one-way coupled approach was used in this research, due to a lower calculation time for the simulation of particle motion within complex geometries in a highly inhomogeneous magnetic field and with an inhomogeneous fluid flow field.

\section{Material and Methods}

The geometries constructed in this study were made with the Computer Aided Design (CAD) software $\mathrm{NX}^{\mathrm{TM}} 8$ 
from Siemens AG in a 1:1 scale. In Figure 1(a) an exploded drawing of the magnetic filter is shown (patent pending with reference number DE102012023382.5). The fluid enters the filter through the inlet, is moving through a flow homogenisator and the following filter matrix and leaves the filter through the outlet. The magnetic circuit of the separator is closed by the tapered surfaces on the XZ-plane of the filter chamber. The filter matrix is placed in the middle of the filter chamber and fixed with a needle construction.

Between the filter plates of the filter matrix a highly inhomogeneous magnetic field with magnetic flux density gradients at the corners of the matrix filaments is generated. The filter is constructed for a placement of quartz sight glasses. Herewith optical checks can be performed during filtrations. In Figure 1(b) the inlet area with length information in Z-direction is shown. The inlet has a length of $20 \mathrm{~mm}$ and within this distance the cross-section of the interior gradually becomes rectangular (with X by Y equal to $26 \mathrm{~mm}$ by $18 \mathrm{~mm}$ ) from circular (with a diameter of $8 \mathrm{~mm}$ ). After $30 \mathrm{~mm}$ of the flow homogenisator (see below) the filter chamber begins. The filter matrix bottom edge starts after $24 \mathrm{~mm}$ within the filter channel.

The CAD models were imported in a step 214 format into the simulation software Comsol Multiphysics ${ }^{\circledR}$ 4.3a. Within the simulation software the fluid flow with particles and the magnetic field were calculated. The magnetic field and the fluid flow were calculated stationary. The particle motion was calculated time dependent. Hereby water was used as the fluid with standard properties at room temperature. The magnetic flux densities were measured at the magnetic pole surfaces and then used for an input of the magnetic field simulation. In the following the equations are shown which are used by the software Comsol Multiphysics ${ }^{\circledR} 4$.3a. For further reading a look at the user's guide of Comsol Multiphysics ${ }^{\circledR}$ 4.2a is advised. The Interface "Magnetic Fields, No Currents" in the software Comsol uses the equations 1 to 4 for modeling of magnetostatics in the absence of electric currents, with $\mu_{0}=$ vacuum permeability, $\mu_{R}=$ relative permeability, $V_{m}=$ magnetic potential, $\boldsymbol{H}=$ magnetic field, $\boldsymbol{B}=$ magnetic flux density and $\boldsymbol{n}=$ surface normal.

$$
\begin{gathered}
-\nabla \cdot\left(\mu_{0} \mu_{R} \nabla V_{m}\right)=0 \\
\boldsymbol{H}=-\nabla V_{m} .
\end{gathered}
$$

Furthermore at the boundaries with an inward magnetic flux density $B_{n}$ the Equation (3) was set.

$$
-\boldsymbol{n} \cdot \boldsymbol{B}=B_{n} .
$$

On the rest of the boundaries magnetic shielding was set with the Equation (4).

$$
\boldsymbol{n} \cdot \boldsymbol{B}=0 .
$$

For the fluid field simulation the Navier-Stokes equations were used. The continuity equation (see Equation (5)) describes the conservation of mass for the incompressible fluid. Equation (6) represents the momentum conservation of the laminar simulation. Hereby $\rho=$ density, $\boldsymbol{u}=$ linear velocity, $p=$ pressure, $v=$ dynamic viscosity, $\boldsymbol{F}=$ Force, $U_{0}=$ average linear inlet velocity, $\boldsymbol{I}=$ unit vector and $T$ is used as an indicator for matrix transposition.

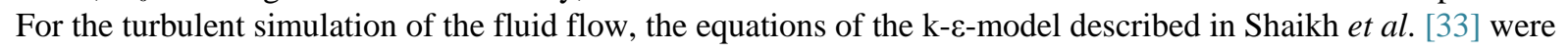
used.

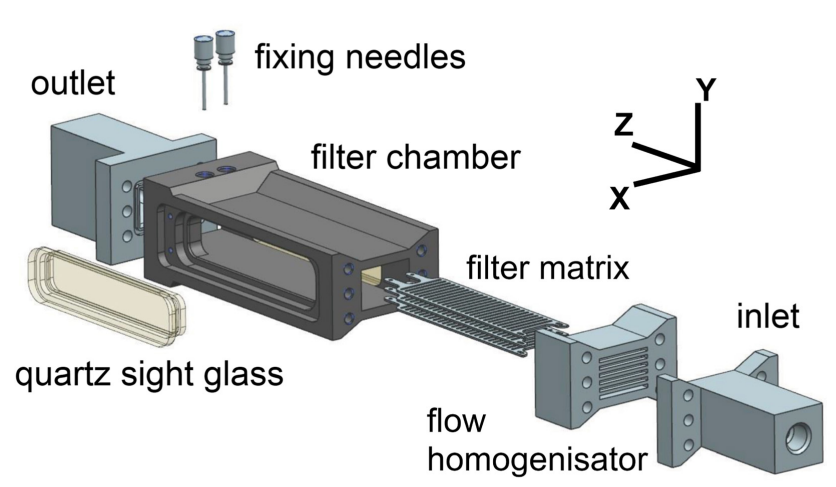

(a)

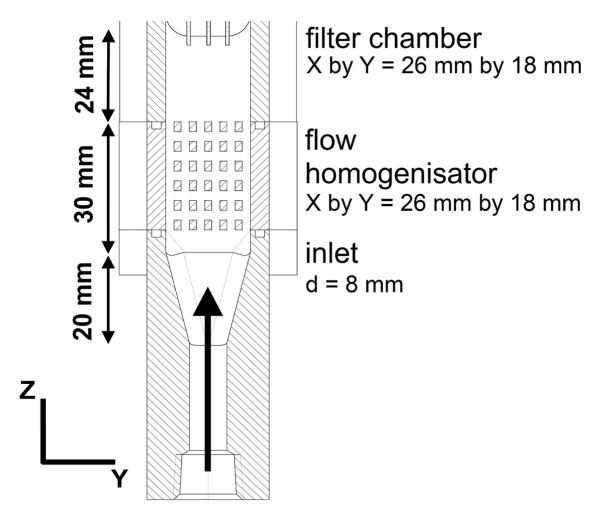

(b)

Figure 1. Constructed and manufactured filter for magnetic separation ((a) patent pending with reference number DE102012023382.5) and YZ-cross section of the inlet area (b). 


$$
\begin{gathered}
\rho \nabla \cdot \boldsymbol{u}=0 \\
\rho(\boldsymbol{u} \cdot \nabla) \boldsymbol{u}=\nabla \cdot\left[-p \boldsymbol{I}+v\left(\nabla \boldsymbol{u}+(\nabla \boldsymbol{u})^{\mathrm{T}}\right)\right]+\boldsymbol{F} .
\end{gathered}
$$

At the boundaries the velocity was zero for the laminar simulation. The inlet boundary condition is represented by Equation (7).

$$
\boldsymbol{u}=U_{0} \cdot \boldsymbol{n} .
$$

The time dependent motion of the virtual particles is described by Equations (8) to (10), whereby $m_{P}=$ mass of the particle, $\boldsymbol{v}=$ linear particle velocity, $\boldsymbol{F}_{D}=$ drag force, $\boldsymbol{F}_{M}=$ magnetic force and $M=$ specific saturation magnetization.

$$
\begin{gathered}
\frac{\partial}{\partial t}\left(m_{p} \boldsymbol{v}\right)=\boldsymbol{F}_{D}+\boldsymbol{F}_{M} \\
\boldsymbol{F}_{D}=\left(\frac{18 v}{\rho_{p} d_{p}^{2}}\right) m_{p}(\boldsymbol{u}-\boldsymbol{v}) \\
\boldsymbol{F}_{M}=\nabla \boldsymbol{B} \cdot M \cdot m_{p} .
\end{gathered}
$$

It was assumed that the calculated amount of particles at the filter outlet, which is only accurate for low particle concentrations, provides information on the performance of the magnetic filter. When more and more particles attach to these primary deposits during the filtration, a filter clogging arises and CFD/Discrete Element Method modeling is required to describe the process. This approach was previously studied by Lindner et al. [30]. Hereby the amount of simulated particles is a parameter, which limits the correlation to the measured results. The time and power which is required to calculate such results becomes very high. This is a reason why this kind of simulation is limited to simple geometries and small amounts of particles and consequently not used here.

The experiments for the evaluation of the simulation results were carried out with a high-gradient magnetic separation laboratory plant which consists of a magnetic separator, a pump and a flow meter. The devices are controlled by a process control system realized with National Instruments ${ }^{\circledR}$ LabView $^{\text {TM }} 8$. The peristaltic pump of the type Ismatec ${ }^{\circledR}$ MCP from the company IDEX Health \& Science GmbH is equipped with a pumphead type Easy-Load 2 from the company Masterflex ${ }^{\circledR} \mathrm{SE}$, whereby the pump tubing is C-Flex with an inner diameter of $4.8 \mathrm{~mm}$. The tubing of the pilot plant is realized with polyurethane tubes with an inner diameter of $6 \mathrm{~mm}$ and quick connectors made of polyoxymethylene from the company Riegler \& Co. KG. The high-gradient magnetic separator type HGF10 from the company Steinert Elektromagnetbau GmbH is equipped with N42 permanent magnets, which can be rotated by an electrical motor. Hereby the magnetic field within the magnetic yoke can be switched on and off [11] [19]. The measurement of the magnetic field within the air gap of the magnetic yoke was carried out with a Gaussmeter type BGM101 from the company Brockhaus Messtechnik GmbH \& Co. KG. The filter housing is made of stainless steel (AISI 316Ti) and consists of an in- and an outlet and the filter chamber. The plates of the filter matrix are made of stainless steel plates (AISI 430) with a thickness of $0.75 \mathrm{~mm}$ by micro waterjet cutting and electro polishing.

The polyvinyl alcohol based magnetizable particles (M-PVA magnetic beads) used in this study, were produced by PerkinElmer chemagen Technologie GmbH with a polydispersed diameter distribution ranging from $<1$ $\mu \mathrm{m}$ to $10 \mu \mathrm{m}$. The particles consisted of equal proportions of magnetite and polyvinyl alcohol and had a dry density of $2 \mathrm{~kg} \cdot \mathrm{dm}^{-3}$. The particle concentration in the filtrate was measured at the outlet of the magnetic filter with an UV/VIS spectrometer Ultrospec 2100 pro from the company GE Healthcare equipped with a flow through cell. The measurement is explained in detail in Shaikh et al. [33].

For the manufacturing of flow homogenisator prototypes, 3D-printing was used with the printer types Objet Eden260VS (material VeroClear-RGD810 for flow homogenisator 1 - 4) and Mojo (material ABSplus for flow homogenisator 5) from the company Stratasys ${ }^{\odot}$ Ltd.

\section{Results}

\subsection{Analysis and Modification of the Magnetic Separator}

For the optimization of the magnetic field between the magnetic pole surfaces, the flux density was measured 
and simulated. By connecting north- and south-pole plates (stainless steel AISI 430) tightly to the yoke, the air gap volume was decreased and the magnetic flux density could be increased (see Figure 2). The filter plates of the filter matrix used in this study had an area of $\mathrm{X}$ by $\mathrm{Z}$ equal to $26 \mathrm{~mm}$ by $80 \mathrm{~mm}$. But the area of the original pole surfaces is $80 \mathrm{~mm}$ by $100 \mathrm{~mm}$ (see Figure 2(b)). Herewith the surface of the poles was nearly 4-times greater than the matrix. In order to obtain identical surface dimensions, the north- and south-pole plates were modified (see Figure 2(c)).

In Figure 3 the measured and simulated magnetic flux densities on a Z-and a X-cutline running through the center of the air gap of the magnetic yoke are shown for the three variations. It can be seen that simulated and measured results are in good correspondence. Furthermore the magnetic flux density could be increased by modifying the magnetic pole plates.

The force acting on a magnetic particle is dependent inter alia on the gradient of the magnetic flux density. It can be seen that the curves in Figure 3 have a great gradient at both endings for the yoke variants B and C. This means that at the edges of the magnetic pole plates the force is at its strongest. The magnetic force acting in an $80 \mathrm{~mm}$ by $27 \mathrm{~mm}$ by $18 \mathrm{~mm}$ volume around the filter matrix of 8 stacked filter plates, which had a thickness of $0.75 \mathrm{~mm}$ and square cutouts, was used for a comparison of yoke B and yoke C. Therefore the integrated magnetic force $\left[\mathrm{nN} \cdot \mathrm{mm}^{3}\right]$ within the volume was calculated for a $3 \mu \mathrm{m}$ magnetic particle with a wet density of 1200 $\mathrm{kg} \cdot \mathrm{m}^{-3}$ and a magnetization of $40 \mathrm{~A} \cdot \mathrm{m}^{2} \cdot \mathrm{kg}^{-1}$. This value was divided by the value of the volume around the matrix. Hereby the average magnetic force in the filter volume was calculated as a scalar value. When the matrix was placed in the yoke B this force was $0.12265 \mathrm{nN}$ and for yoke $C$ it was $0.12881 \mathrm{nN}$ respectively. With the modified pole plate the average magnetic force could be increased by $5 \%$.

In a simulation with a varied magnetic pole plate thickness of type B, it was found that the magnetic flux density in the center of the air gap rose exponentially with a decreasing distance. The distance of the pole surfaces was varied from $s=5 \mathrm{~mm}$ to $40 \mathrm{~mm}$. Equation (11) calculates the magnetic flux density $B$ with the distance $s$ in millimeters (with $R^{2}=0.95$ ).

$$
B[T]=0.8873 \cdot \mathrm{e}^{-0.036 \cdot s} .
$$

For the magnetic pole plates of type $\mathrm{C}$ with a distance of $26 \mathrm{~mm}$, the tapering was varied in another simulation with a final surface ranging from $10 \mathrm{~mm}$ by $10 \mathrm{~mm}$ to $70 \mathrm{~mm}$ by $90 \mathrm{~mm}$. The calculation revealed that for this case no significant changes of the magnetic flux density occur in the center of the air gap. In summary the distance of the pole surfaces to each other has the greatest impact on the maximum of the magnetic flux density in the air gap. But the magnetic flux density gradients at the edges of the surfaces represent the areas with the magnetic force maxima. In this context the reduction of the pole surface area increase the magnetic force within the filter chamber.

\subsection{Analysis and Optimization of the Inlet Fluid Flow}

The inlet of the magnetic filter was constructed in one piece with a flange connection to the filter chamber (see Figure 1). The fluid flows into the filter chamber through the inlet with a circular cross section and a diameter of $8 \mathrm{~mm}$. Then the cross section becomes gradually rectangular with $\mathrm{X}$ by $\mathrm{Y}$ equal to $26 \mathrm{~mm}$ by $18 \mathrm{~mm}$ side lengths. The parallel surfaces in $18 \mathrm{~mm}$ distance represent the pole surfaces within the filter chamber. The outlet was

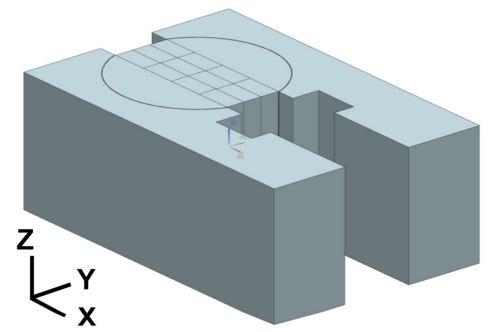

(a)

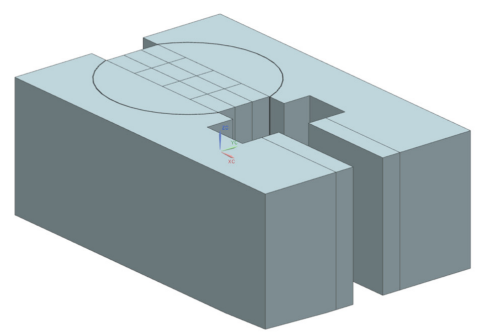

(b)

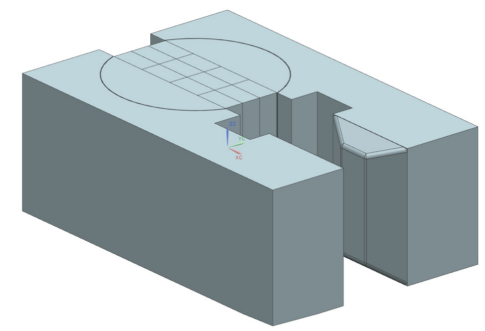

(c)

Figure 2. Variations of the pole plates of the high-gradient magnetic separator type Steinert HGF10 (a) = without plates, (b) = with original plates, $(c)=$ with modified plates. 

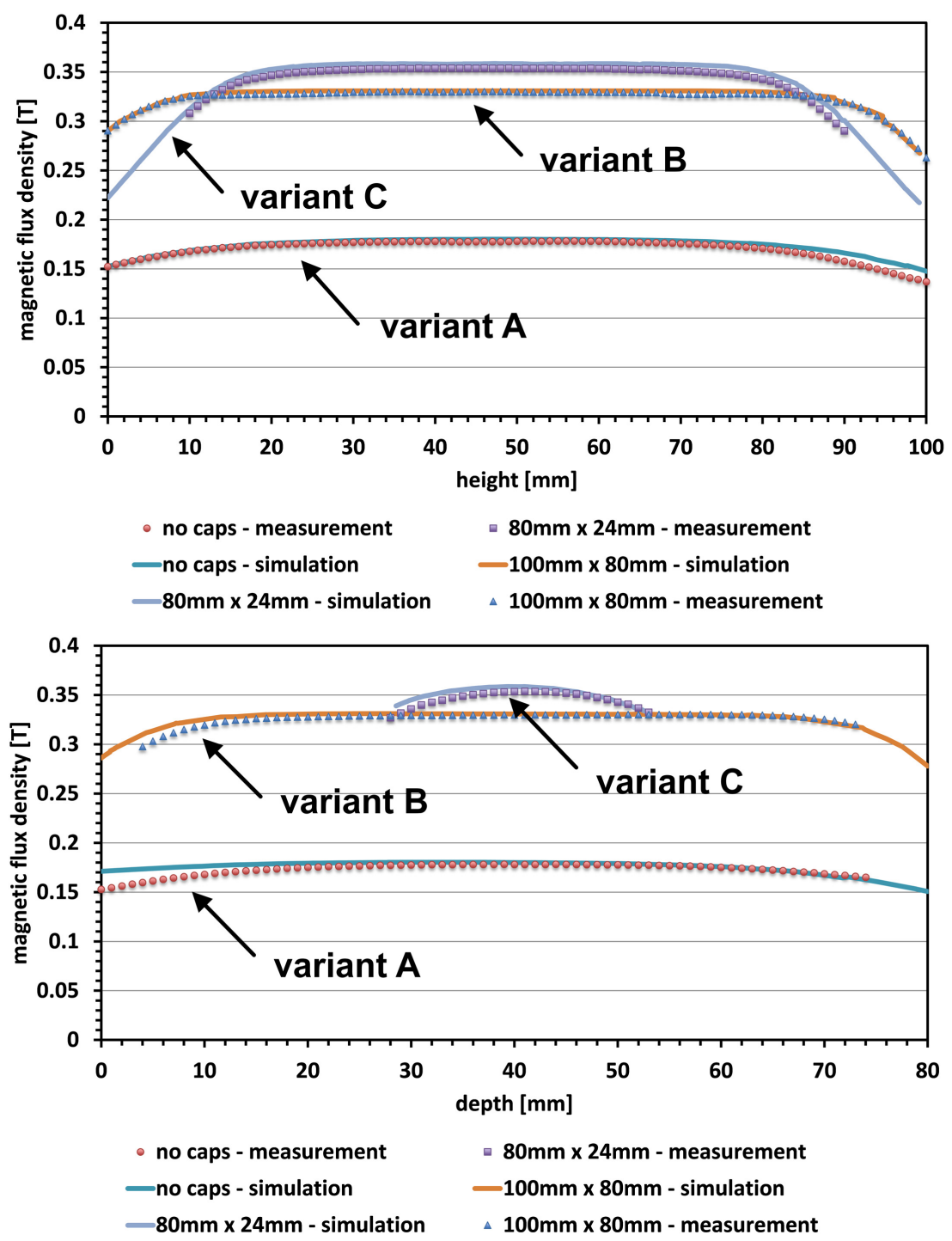

Figure 3. Simulated and measured magnetic flux densities at a Z-cutline (left) and a $\mathrm{X}$-cutline (right) for the three variants of the pole plates (A-C) of Figure 2.

identical to the inlet. The transition area from circular to rectangular could be produced with a maximum length of $20 \mathrm{~mm}$ in Z-direction, due to the milling cutter diameter of $8 \mathrm{~mm}$. Herewith the linear fluid velocity is highly inhomogeneous at the start of the filter matrix. The fluid has a high linear velocity in the center of the cross section. At the boundary areas the velocity is low. This means that the particles have a higher velocity in the areas where the magnetic force is the lowest and vice versa in the front part of the filter matrix. So the deposits of the magnetic particles build up inhomogeneously.

In order to obtain information on how the velocity maximum changes with a varying milling depth, a set of fluid flow simulations were carried out. The length of the transition part was varied from $10 \mathrm{~mm}$ over $20 \mathrm{~mm}$ to $30 \mathrm{~mm}$, while the volume flow was set to $100 \mathrm{~mL} \cdot \mathrm{min}^{-1}$. The maximum linear fluid velocities at the end of the inlet were $59 \mathrm{~mm} \cdot \mathrm{s}^{-1}, 48 \mathrm{~mm} \cdot \mathrm{s}^{-1}$ and $43 \mathrm{~mm} \cdot \mathrm{s}^{-1}$ respectively. When the volume flow was adjusted to 400 $\mathrm{mL} \cdot \mathrm{min}^{-1}$ the maximum flow velocities and slopes were nearly 4-fold greater. The fluid flow profile has a parabolic shape at the center of the cross section. So a solution for the inlet problem could be an increased inlet length. But due to the limitations in manufacturing and otherwise rising costs, this was not further pursued. Additionally, an enlarged inlet would increase the mean residence time of the particles, which was also not favored. In order to homogenize the velocity profile of the fluid flow the installation of a flow homogenisator was re- 
garded as a better solution due to the compact geometry and simple manufacturability. The flow homogenisator length in Z-direction was adjusted to $24 \mathrm{~mm}$. Within this piece of rectangular channel, crossbars with a length of $26 \mathrm{~mm}$ in X-direction were placed, so that the fluid was divided into the direction to the magnetic pole surfaces (see Figure 1).

In a first laminar simulation the amount of crossbars was varied in Y-and Z-direction. In Y-direction the amount of crossbars was adjusted up to 5 (with a thickness of $1.5 \mathrm{~mm}$ ) and in Z-direction up to 6 . In X-direction the length of the crossbars was $26 \mathrm{~mm}$, which was identical to the filter chamber length in X-direction. The height of each crossbar in Z-direction was calculated by the ratio between the sum of crossbar heights and the length of the flow homogenisator $(24 \mathrm{~mm})$. Hereby quotient values of $0.25,0.5$ and 0.75 were adjusted. The ratio calculation was carried out with Equation (12). Hereby $r=$ ratio, $d_{A}=$ distance between crossbars in Z-direction, $d_{C}=$ length of crossbars in Z-direction. $d_{C}$ and $d_{A}$ are identical when the ratio is adjusted to $r=0.5$. $d_{C}$ has a higher value than $d_{A}$ in case of $r=0.75$ and vice versa in the case of $r=0.25$.

$$
r=\frac{\sum d_{C}}{\sum d_{A}+\sum d_{C}}
$$

The first prototype of the geometry was manufactured via 3D-printing with 5 horizontal and 6 vertical crossbars with a ratio of 0.5 (see Figure 4).

In Figure 5 the simulation results for the linear fluid velocities, which develop $5 \mathrm{~mm}$ after the flow homogenisator, are shown for an ingoing volume flow of $100 \mathrm{~mL} \cdot \mathrm{min}^{-1}$ on two crossbred $\mathrm{X}$ - and Y-cut lines. These lines are running through the center of the rectangular filter channel. For a clear presentation the pictured results were limited to the following combinations: $\mathrm{h}=2$ and 6 (amount of horizontal crossbars), $\mathrm{v}=2,4$ and 6 (amount of vertical crossbars), $r=0.25,0.5$ and 0.75 (ratio of crossbar height). The abscissa in Figure 5 represents the halved cut lines due to volume symmetry. The ordinates show the linear fluid velocity. It can be seen that primarily the amount of horizontal crossbars have an impact on the maximum linear velocity after the flow homogenisator. The amount of vertical crossbars and the height of the crossbars had a minor impact on the fluid velocity. Therefore the division of the fluid flow in Y-direction by a high number of crossbars $(\mathrm{h}=6)$ mainly influences the fluid profile. Only two rows of crossbars in Z-direction $(v=2)$ and a ratio of $r=0.25$ are sufficient to homogenize the flow so that the linear fluid velocity was nearly identical over the cross section. Within this context the Bernoulli Effect is a reason for the homogenization of the fluid profile with a high number of horizontal crossbars. Between the crossbars the linear fluid velocity increases and after an array of crossbars the velocity overall decreases again while the fluid is mixed.

Magnetic filtrations with threefold repetition were carried out with the manufactured flow homogenisator to check its effect. A dense magnetic particle suspension with a concentration of $5 \mathrm{~g} \cdot \mathrm{L}^{-1}$ was filtrated with a volume flow of $100 \mathrm{~mL} \cdot \mathrm{min}^{-1}$ by an exemplary matrix of eight stacked filter plates. In Figure 6 the at-line measured mean particle concentration in the filtrate is plotted on the ordinate and the time dependent cumulative particle

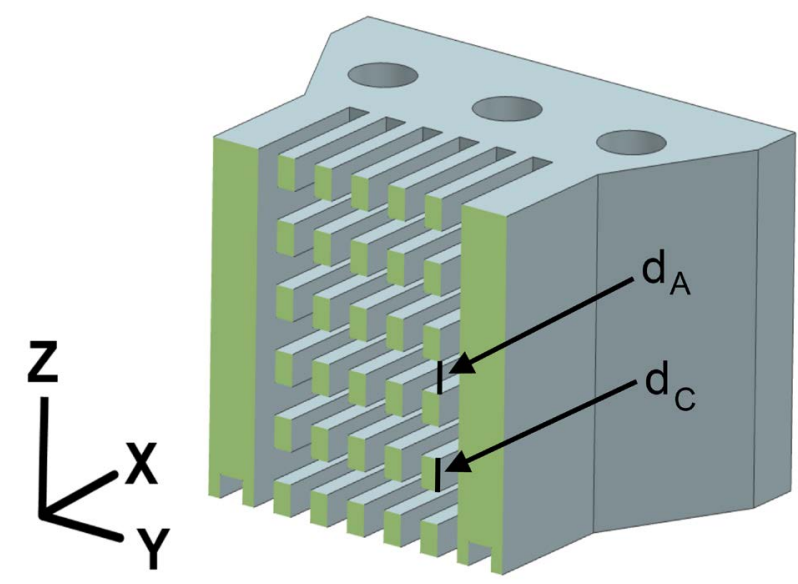

Figure 4. Symmetrical cross section of the flow homogenisator prototype type 1. 

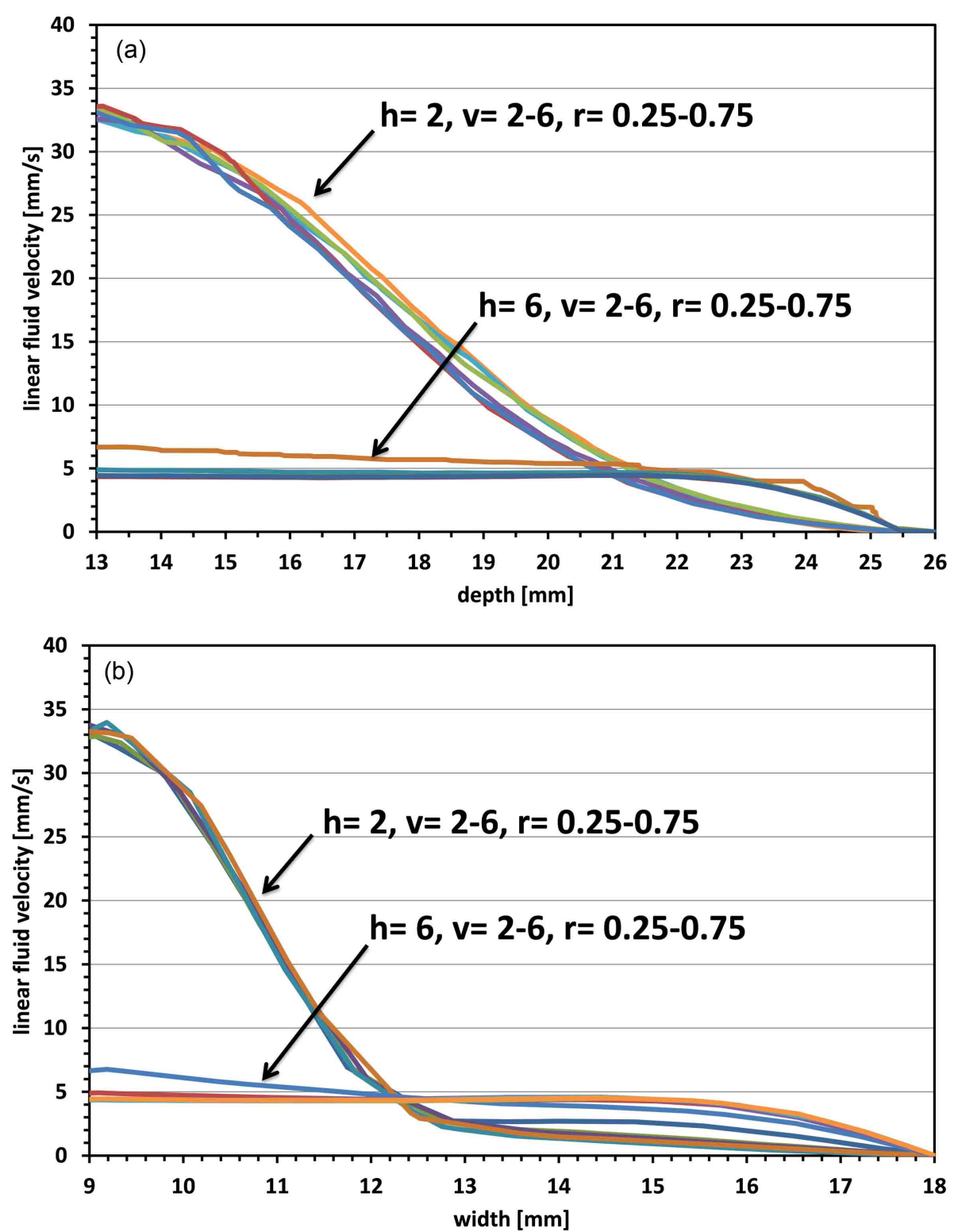

Figure 5. Calculated linear fluid velocity on X-cutline (a) and Y-cut line (b) running through the center of the filter channel $5 \mathrm{~mm}$ after the flow homogenisator.

inlet mass on the abscissa. Furthermore the standard deviation for each data point is plotted as a gray colored error bar. The filtration with the flow homogenisator shows the desired more evenly development. In the range from $3.5 \mathrm{~g}$ to $4 \mathrm{~g}$ cumulative particle inlet mass the filtration without the flow homogenisator is clearly worse than with a flow homogenisator. In both cases a filter breakthrough could be measured, but in case of a flow homogenisator the breakthrough appeared after $4.3 \mathrm{~g}$ of particle input rather than $3.5 \mathrm{~g}$. It was assumed the filter clogging was built up more evenly by using a flow homogenisator. Herewith the amount of re-released particle fractions from the filter clogging was lower than in case without a flow homogenisator. So the experiments confirm the simulated results.

In a further simulation study supplemental flow homogenisator geometries were simulated in terms of the particle retention. The variation was focused on the crossbar constellation. The crossbars were aligned in two patterns: 1) behind and 2) shifted to each other (see Figure 7). The second type of constellation has the advantage that the fluid could not flow linearly through the flow homogenisator. The flow homogenisator type 5 was 


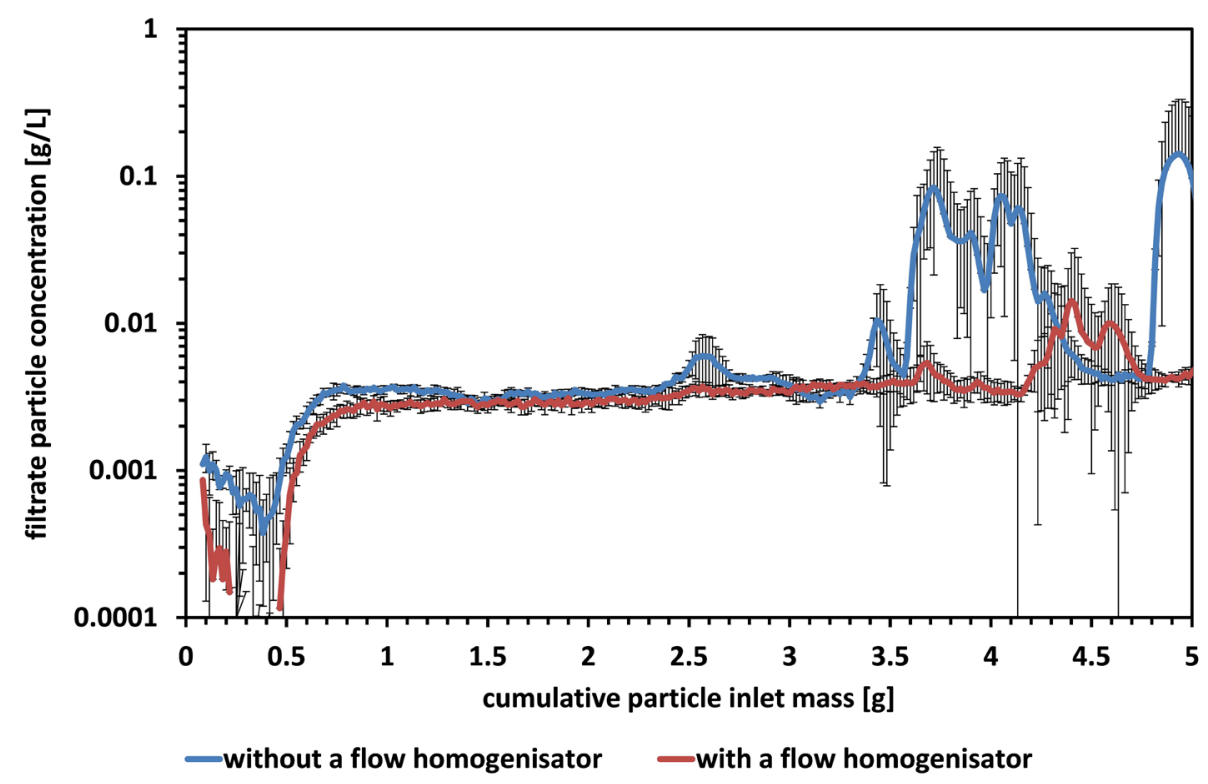

Figure 6. Measured filtrate particle concentration for filtrations with and without a flow homogenisator (threefold repetition for calculation of mean value and standard deviation as gray colored error bars).
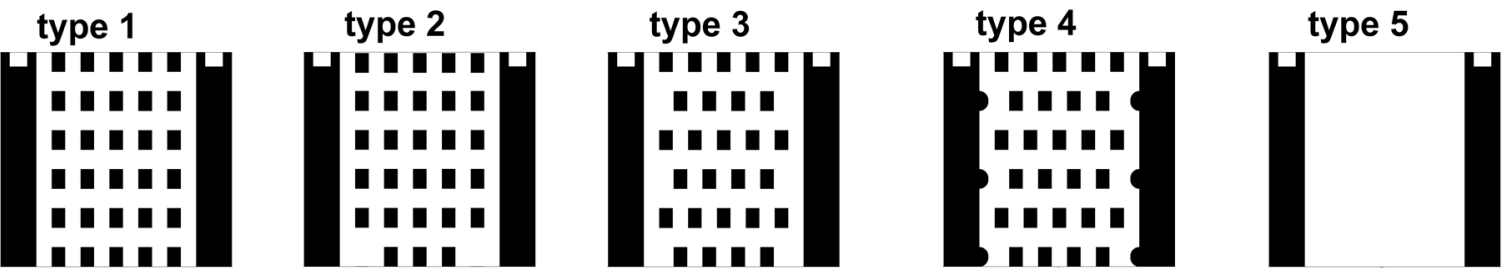

Figure 7. Cross section of the flow homogenisator types 1 - 5.

introduced to analyze the flow velocity without crossbars.

The turbulent volume flow of water was adjusted to $400 \mathrm{~mL} \cdot \mathrm{min}^{-1}$.Within this simulation 10,000 virtual magnetizable particles were used with a uniformly distributed diameter from $0.2 \mu \mathrm{m}$ to $10.2 \mu \mathrm{m}$. The magnetization was adjusted to $40 \mathrm{~A} \cdot \mathrm{m}^{2} \cdot \mathrm{kg}^{-1}$ and the release of the particle was located at the inlet of the filter. After a simulation time frame of $60 \mathrm{~s}$ the amount of particles at the outlet was counted and used for the ratio calculation between the amounts of particles flowing in and out.

An experimental setup, which was very similar to the simulation conditions, was used to measure the filtrate particle mass in real filtrations. Therefore $1 \mathrm{~mL}$ of a particle suspension with a concentration of $20 \mathrm{~g} \cdot \mathrm{L}^{-1} \mathrm{was}$ injected with a syringe into the tubing at the inlet of the magnetic filter, while the feed pump was out of action. The particles used for the experiments had a median diameter of $5.4 \mu \mathrm{m}$ but a significantly lower amount of small size particles (with a diameter smaller than $2 \mu \mathrm{m}$ ) in comparison to the particle size distribution in the simulation. After injection the pump was started with a volume flow of $400 \mathrm{~mL} \cdot \mathrm{min}^{-1}$. To ensure a complete particle run-through with an expected mean residence time of $20 \mathrm{~s}$, the pumping was applied for $240 \mathrm{~s}$. At the outlet of the filter chamber the concentration of particles was measured according to Shaikh et al. [33] and used for the ratio calculation similar to the simulation. Figure 8 shows the particle retention results of the experiments (Figure 8(a)) and the results of the simulations (Figure 8(b)) as bar charts.

It can be seen that the amount of particles in the filtrate is significantly higher in the simulation than in real filtrations. During high-gradient magnetic separation the impact of the drag force on smaller particles is greater than the impact of the magnetic force. This means that smaller particles could pass the magnetic filter easier. Consequently the median particle diameter of the particles in the experiment, which passed the magnetic filter, was below $3.56 \mu \mathrm{m}$ in all cases (in comparison to $5.4 \mu \mathrm{m}$ in the feed). Therefore the difference between calculations and experiments can be explained with the different particle size distributions. But the simulations as well 

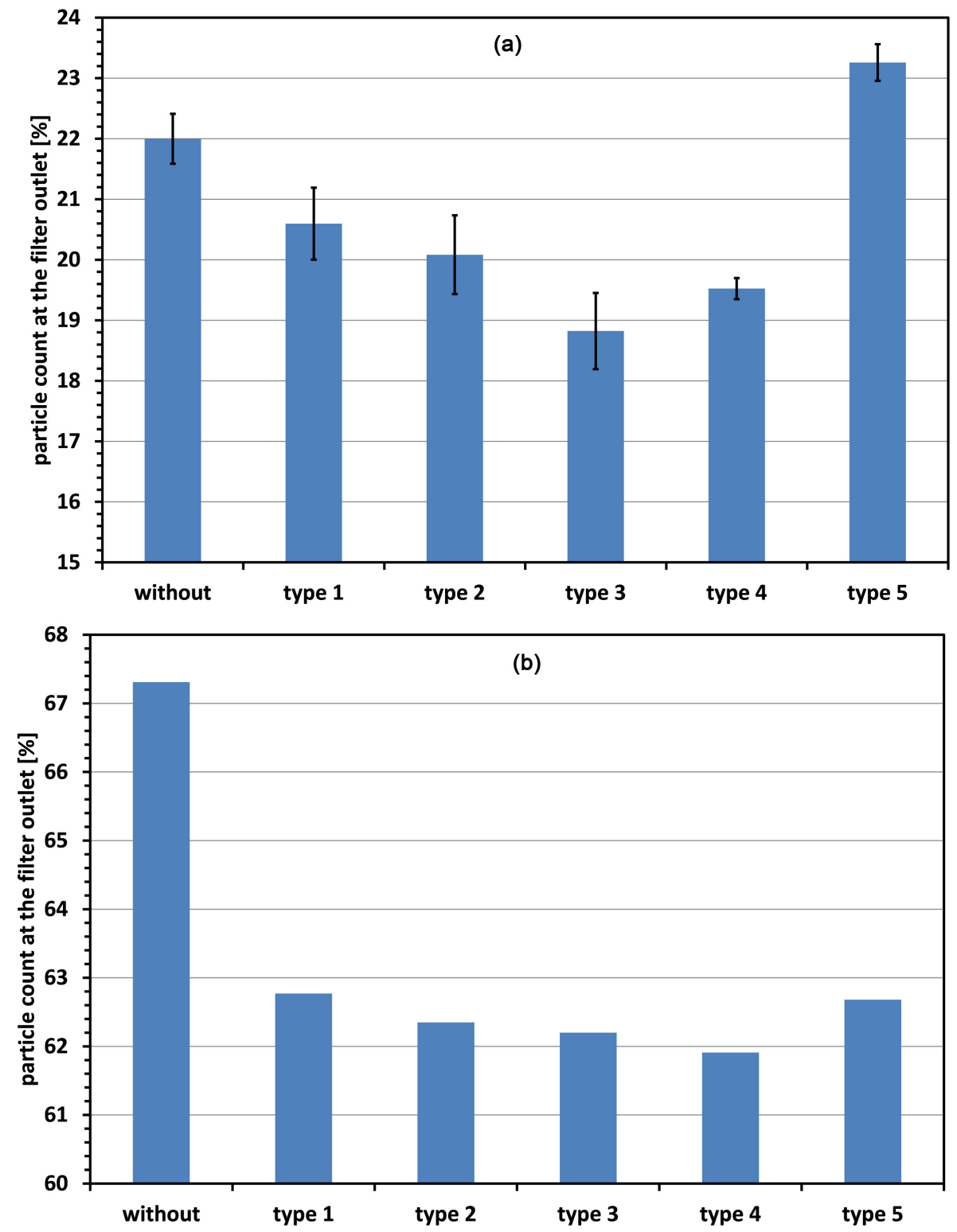

Figure 8. Measured (a) and calculated (b) amount of particle at the outlet of the filter with flow homogenisator types 1 - 5 .

as the experiments pointed out that the flow homogenisator types 3 and 4 had the best performance in particle retention.

Interestingly, the highest amount of particles at the outlet of the filter was measured in the experiment with the flow homogenisator type 5 (see Figure 8(a)). In contrast to the 3D-printing of the flow homogenisator types 1 to 4 (resolution $85 \mu \mathrm{m}$ ) the 3D-printing of type 5 was done in a lower printing resolution of $254 \mu \mathrm{m}$ with a higher surface roughness but an increased hardness. The increased hardness was required because of the lack of stabilizing crossbars. The higher surface roughness causes an increased turbulence in the filter chamber which leads to a reduced separation. Due to the fact that this is not properly included in the simulation the discrepancy between simulation and measurement arose regarding the effect of flow homogenisator type 5.

\subsection{Influence of the Geometry of the Filter Plates}

After finding an optimum geometry of the flow homogenisator crossbars, the research was continued with an 
analysis of the particle retention on filter matrices with cutouts. By cutting out pattern in the filter plates, the magnetic flux density field becomes strongly inhomogeneous and at the corners of the cutouts the magnetic force maxima build up.

The time dependent simulation of particle retention on a filter matrix, consisting of 8 stacked filter plates with a thickness of $0.75 \mathrm{~mm}$, a X by Z dimension of $26 \mathrm{~mm}$ by $80 \mathrm{~mm}$ and a relative permeability of 1000 was performed with a laminar volume flow of $200 \mathrm{~mL} \cdot \mathrm{min}^{-1}$. The amount of virtual magnetizable particles was adjusted to 1000 particles with a wet density of $1200 \mathrm{~kg} \cdot \mathrm{m}^{-3}$. The particle diameter was uniformly distributed between $0.2 \mu \mathrm{m}$ and $10.2 \mu \mathrm{m}$. After a simulation time frame of $45 \mathrm{~s}$ the amount of particles at the outlet was counted. Furthermore the motion of non-magnetizable particles, which could retain on the matrix due to hindrance, was also simulated and used for the ratio calculation.

The plates of the filter matrix inserted into the center of the filter chamber had different plate patterns. The design of the cutouts was matched to result in a constant plate volume of $870 \mathrm{~mm}^{3}$. For reference a plate without cutouts and a plate volume of $1440 \mathrm{~mm}^{3}$ was used. In Figure 9(a) the geometries of the filter plates are shown. Figure 9(b) shows a plot with the calculated amount of particles at the outlet of the filter on the ordinate and the simulation time on the abscissa. It can be seen that the particle amount at the outlet is the highest in case of filter plates with no cutouts. The circular and square cutouts were also not suitable for the magnetic filtration. By comparison the length- and widthwise cutouts had the lowest particle amount at the outlet in the simulation and seemed to be generally suitable. But in practice a problem occurs by using the lengthwise filter plates when the magnetic field is switched on. The long filaments bend due to magnetic forces and form a deformed matrix. Therefore widthwise cutouts were regarded as optimum.

The total edge length of all corners of a filter plate could be identified as the major parameter, which affects the particle retention. The total edge lengths of the filter plates (widthwise: $2582 \mathrm{~mm}$, circular: $1924 \mathrm{~mm}$, square: $2084 \mathrm{~mm}$, lengthwise: $2589 \mathrm{~mm}$, without: $416 \mathrm{~mm}$ ) are in good accordance to the simulated retention performances of the filter plates.

The analysis of the median particle diameter at the outlet of the magnetic filter showed that the magnetizable particles had different $\mathrm{d}_{50}$ values (widthwise: $0.89 \mu \mathrm{m}$, circular: $6.21 \mu \mathrm{m}$, square: $5.96 \mu \mathrm{m}$, lengthwise: $0.69 \mu \mathrm{m}$, without: $5.56 \mu \mathrm{m}$ ). In all simulations the non-magnetizable particles at the outlet of the magnetic filter had the same median particle diameter of $5.4 \mu \mathrm{m}$. In case of the simulation of width- and lengthwise cutouts only very small magnetizable particles flow through the magnetic filter in comparison to the median particle diameter of the ingoing particles $\left(d_{50}=5.2 \mu \mathrm{m}\right)$. This shows again the good retention performance of those two geometries.

For an experimental testing of the filter matrix geometry, filtrations with filter matrices of 8 stacked filter plates, each with either widthwise or square cutouts, were carried out. The suspension had a particle concentration of $5 \mathrm{~g} \cdot \mathrm{L}^{-1}$ and the volume flow was adjusted to $100 \mathrm{~mL} \cdot \mathrm{min}^{-1}$. In Figure 10 the measured mean particle

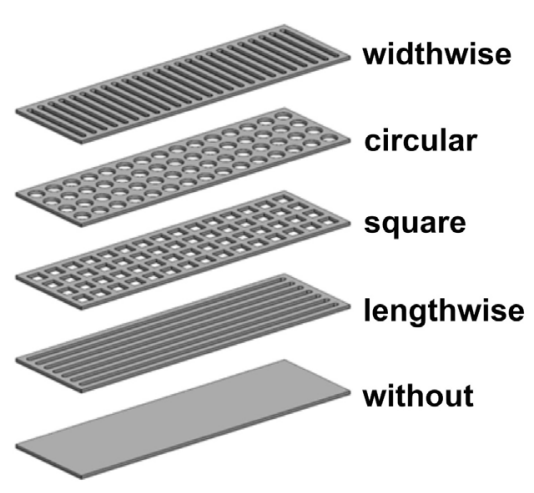

(a)

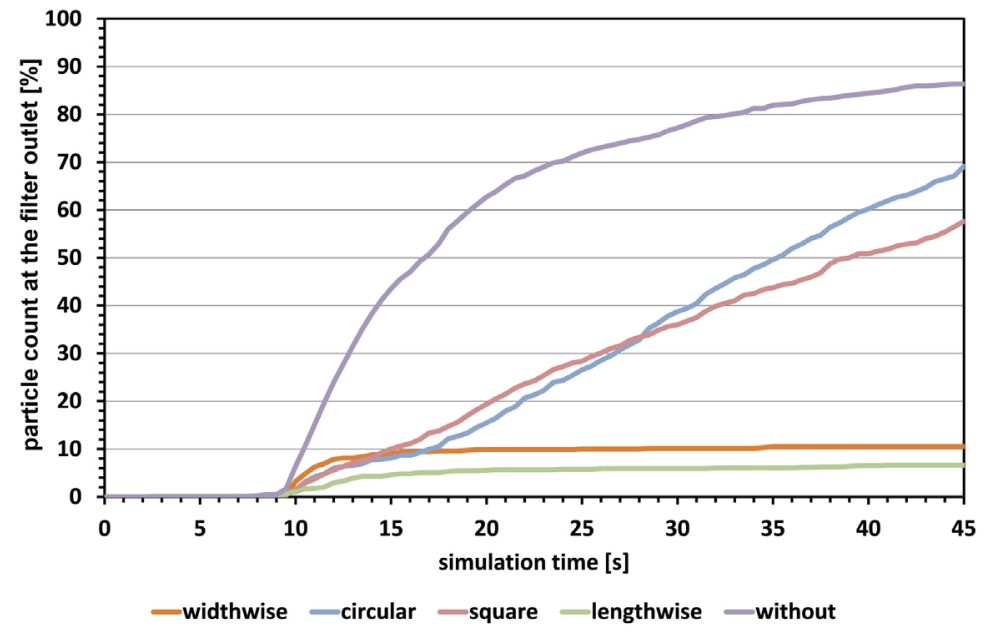

(b)

Figure 9. Filter plate pattern (a) and calculated amount of particles at the outlet of the filter with different filter matrices consisting of 8 stacked filter plates (b). 


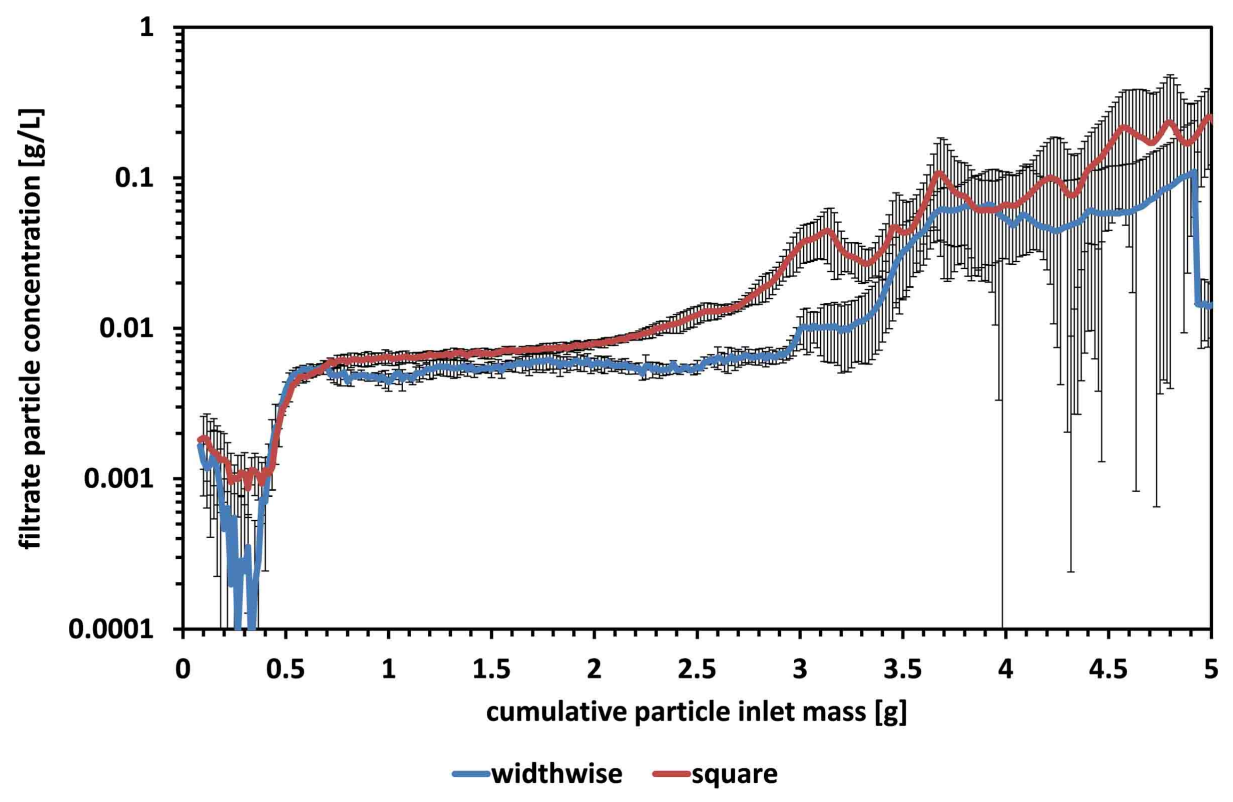

Figure 10. Measured particle concentration in the filtrate with a filter matrix consisting of 8 stacked filter plates with either widthwise or square cutouts (see Figure 9).

concentration (including gray colored error bars) in the filtrate is plotted along with the time dependent cumulative particle inlet mass.

After a total inlet of $2 \mathrm{~g}$ magnetizable particles the particle concentration in the filtrate begins to rise significantly in case of square cutouts. At this point the retention within the filter begins to decrease. The use of widthwise cutouts results in a constant retention up to $3 \mathrm{~g}$ of magnetizable particles, which represents a retention improvement of $50 \%$.

\section{Summary and Conclusion}

Computational methods to study the effects of geometric variations of magnetic filters have a great potential to reduce time and costs. The one-way coupled Langrangian approach represents a simple way to calculate the particle motion within the magnetic field and the fluid flow field. The geometry of the filter matrix should have as much as possible total edge length to maximize the particle retention.

It could be shown that the inlet profile of the fluid flow had an impact on the retention of magnetizable particles in the filter matrix as well. The linear fluid velocity should be homogenous at the start of the filter matrix, otherwise the particle deposits are built up irregularly on the filter plates. In this case, the concentration of particles in the filtrate rises faster while the magnetic filtration takes place. This means that the retention within the filter decreases with an increasing velocity distribution at the inlet cross section. When a flow homogenisator is installed prior to the filter matrix, the particle retention can be improved and stays constant over a longer filtration time.

\section{Acknowledgements}

The authors acknowledge financial support received from the German Federal Ministry of Education and Research (BMBF) (Project reference No: 0316057B). Furthermore the authors thank Jonas Wohlgemuth, Karlsruhe Institute of Technology, Institute of Functional Interfaces for the 3D-printing of the flow homogenisator prototypes.

\section{References}

[1] Safarik, I. and Safarikova, M. (2004) Magnetic Techniques for the Isolation and Purification of Proteins and Peptides. BioMagnetic Research and Technology, 2, 7. http://dx.doi.org/10.1186/1477-044X-2-7 
[2] Franzreb, M., Siemann-Herzberg, M., Hobley, T.J. and Thomas, O.R. (2006) Protein Purification Using Magnetic Adsorbent Particles. Applied Microbiology and Biotechnology, 70, 505-516. http://dx.doi.org/10.1007/s00253-006-0344-3

[3] Hanefeld, U., Gardossi, L. and Magner, E. (2009) Understanding Enzyme Immobilisation. Chemical Society Reviews, 38, 453-468. http://dx.doi.org/10.1039/B711564B

[4] Homaei, A.A., Sariri, R., Vianello, F. and Stevanato, R. (2013) Enzyme Immobilization: An Update. Journal of Chemical Biology, 6, 185-205. http://dx.doi.org/10.1007/s12154-013-0102-9

[5] Mu, X., Qiao, J., Qi, L., Dong, P. and Ma, H. (2014) Poly(2-vinyl-4, 4-dimethylazlactone)-Functionalized Magnetic Nanoparticles as Carriers for Enzyme Immobilization and Its Application. ACS Applied Materials \& Interfaces, 6, 21346-21354. http://dx.doi.org/10.1021/am5063025

[6] Misson, M., Zhang, H. and Jin, B. (2015) Nanobiocatalyst Advancements and Bioprocessing Applications. Journal of the Royal Society Interface, 12, No. 102. http://dx.doi.org/10.1098/rsif.2014.0891

[7] Setchell, C.H. (1985) Magnetic Separations in Biotechnology-A Review. Journal of Chemical Technology and Biotechnology, 35, 175-182. http://dx.doi.org/10.1002/jctb.280350302

[8] Franzreb, M. (2003) Magnettechnologie in der Verfahrenstechnik wässriger Medien. Wissenschaftliche Berichte FZKA, 6916, A-212.

[9] Augusto, P.A., Castelo-Grande, T. and Augusto, P. (2005) Magnetic Classification in Health Sciences and in Chemical Engineering. Chemical Engineering Journal, 111, 85-90. http://dx.doi.org/10.1016/j.cej.2005.02.013

[10] Yavuz, C.T., Prakash, A., Mayo, J.T. and Colvin, V.L. (2009) Magnetic Separations: From Steel Plants to Biotechnology. Chemical Engineering Science, 64, 2510-2521. http://dx.doi.org/10.1016/j.ces.2008.11.018

[11] Hoffmann, C., Franzreb, M. and Höll, W.H. (2002) A Novel High-Gradient Magnetic Separator (HGMS) Design for Biotech Applications. IEEE Transactions on Applied Superconductivity, 12, 963-966. http://dx.doi.org/10.1109/TASC.2002.1018560

[12] Schultz, N., Syldatk, C., Franzreb, M. and Hobley, T.J. (2007) Integrated Processing and Multiple Re-Use of Immobilised Lipase by Magnetic Separation Technology. Journal of Biotechnology, 132, 202-208. http://dx.doi.org/10.1016/j.jbiotec.2007.05.029

[13] Maury, T.L., Ottow, K.E., Brask, J., Villadsen, J. and Hobley, T.J. (2012) Use of High-Gradient Magnetic Fishing for Reducing Proteolysis during Fermentation. Biotechnology Journal, 7, 909-918. http://dx.doi.org/10.1002/biot.201100376

[14] Ebner, N., Gomes, C.S., Hobley, T.J., Thomas, O.R. and Franzreb, M. (2007) Filter Capacity Predictions for the Capture of Magnetic Microparticles by High-Gradient Magnetic Separation. IEEE Transactions on Magnetics, 43, 19411949. http://dx.doi.org/10.1109/TMAG.2007.892080

[15] Meyer, A., Hansen, D.B., Gomes, C.S., Hobley, T.J., Thomas, O.R. and Franzreb, M. (2005) Demonstration of a Strategy for Product Purification by High-Gradient Magnetic Fishing: Recovery of Superoxide Dismutase from Unconditioned Whey. Biotechnology Progress, 21, 244-254. http://dx.doi.org/10.1021/bp049656c

[16] Pasteur, A., Tippkötter, N., Kampeis, P. and Ulber, R. (2014) Optimization of High Gradient Magnetic Separation Filter Units for the Purification of Fermentation Products. IEEE Transactions on Magnetics, 50, 1-7. http://dx.doi.org/10.1109/TMAG.2014.2325535

[17] Chang, C.F., Chang, C.Y. and Hsu, T.L. (2011) Removal of Fluoride from Aqueous Solution with the Superparamagnetic Zirconia Material. Desalination, 279, 375-382. http://dx.doi.org/10.1016/j.desal.2011.06.039

[18] Ueda, H., Agatsuma, K., Kajikawa, K., Furuse, M., Fuchino, S. and Ishiyama, A. (2009) Design and Test of Filter of High Gradient Magnetic Separation System for Trapping Immunoglobulin in Serum. IEEE Transactions on Applied Superconductivity, 19, 2157-2161. http://dx.doi.org/10.1109/TASC.2009.2018441

[19] Kampeis, P., Bewer, M. and Rogin, S. (2009) Einsatz von Magnetfiltern in der Bioverfahrenstechnik Teil 1: Vergleich verschiedener Verfahren zum Rückspülen der Magnetfilter. Chemie Ingenieur Technik, 81, 275-281. http://dx.doi.org/10.1002/cite.200800121

[20] König, S., Schumann, C. and Kampeis, P. (2010) Einsatz von Magnetfiltern in der Bioverfahrenstechnik-Teil 2: Trennung von Biomasse und Magnetbeads im Magnetfilter. Chemie Ingenieur Technik, 82, 641-650. http://dx.doi.org/10.1002/cite.200900106

[21] Mohanty, S., Das, B. and Mishra, B.K. (2011) A Preliminary Investigation into Magnetic Separation Process Using CFD. Minerals Engineering, 24, 1651-1657. http://dx.doi.org/10.1016/j.mineng.2011.08.021

[22] Zhang, J., Feng, Z., Jia, X., Liang, M., Men, Z., Zhang, Y., Bu, Y. and Li, W. (2013) High Gradient Magnetic Separation of Catalyst/Wax Mixture in Fischer-Tropsch Synthesis: Modeling and Experimental Study. Chemical Engineering Science, 99, 28-37. http://dx.doi.org/10.1016/j.ces.2013.05.005

[23] Menzel, K., Lindner, J. and Nirschl, H. (2011) Treatment of Highly Viscous Lubricants by High Gradient Magnetic 
Separation Technique. FILTECH Conference Proceedings, Vol. I, Wiesbaden, 22-24 March 2011, 103-108.

[24] Mizuno, N., Mishima, F., Akiyama, Y., Okada, H., Hirota, N., Matsuura, H., Maeda, T., Shigemoto, H. and Nishijima, S. (2015) Removal of Iron Oxide with Superconducting Magnet High Gradient Magnetic Separation from Feed-Water in Thermal Plant. IEEE Transactions on Applied Superconductivity, 25, 1-4. http://dx.doi.org/10.1109/TASC.2015.2392624

[25] Furlani, E.P., Sahoo, Y., Ng, K.C., Wortman, J.C. and Monk, T.E. (2007) A Model for Predicting Magnetic Particle Capture in a Microfluidic Bioseparator. Biomedical Microdevices, 9, 451-463. http://dx.doi.org/10.1007/s10544-007-9050-X

[26] Khashan, S.A., Elnajjar, E. and Haik, Y. (2011) CFD Simulation of the Magnetophoretic Separation in a Microchannel. Journal of Magnetism and Magnetic Materials, 323, 2960-2967. http://dx.doi.org/10.1016/j.jmmm.2011.06.001

[27] Khashan, S.A., Haik, Y. and Elnajjar, E. (2012) CFD Simulation for Biomagnetic Separation Involving Dilute Suspensions. The Canadian Journal of Chemical Engineering, 90, 1450-1456. http://dx.doi.org/10.1002/cjce.21639

[28] Villani, D.D., Yung, K.W. and Landecker, P.B. (1998) An Analytic Solution for the Force between Two Magnetic Dipoles. Magnetic and Electrical Separation, 1998, 39-52. http://dx.doi.org/10.1155/1998/79537

[29] Cherry, E.M. and Eaton, J.K. (2014) A Comprehensive Model of Magnetic Particle Motion during Magnetic Drug Targeting. International Journal of Multiphase Flow, 59, 173-185. http://dx.doi.org/10.1016/j.ijmultiphaseflow.2013.11.007

[30] Lindner, J., Menzel, K. and Nirschl, H. (2013) Simulation of Magnetic Suspensions for HGMS Using CFD, FEM and DEM Modeling. Computers \& Chemical Engineering, 54, 111-121. http://dx.doi.org/10.1016/j.compchemeng.2013.03.012

[31] Ravnik, J. and Hriberšek, M. (2013) High Gradient Magnetic Particle Separation in Viscous Flows by 3D BEM. Computational Mechanics, 51, 465-474. http://dx.doi.org/10.1007/s00466-012-0729-3

[32] Khashan, S.A. and Furlani, E. (2011) CFD-Based, Lagrangian-Eulerian Coupling Approach for Magnetophoretic Particle Capture. 3rd Micro and Nano Flows Conference Thessaloniki, Thessaloniki, 22-24 August 2011.

[33] Shaikh, Y.S., Seibert, C., Schumann, C., Ferner, M.J., Raddatz, H. and Kampeis, P. (2016) Optimizing a Rotor-Stator Filter Matrix for High-Gradient Magnetic Separation of Functionalized Magnetic Particles. Engineering in Life Sciences. http://dx.doi.org/10.1002/elsc.201500115 